Research Article

\title{
Modifications and Statistical Analysis of Acoustic Emission Models Based on the Damage and Fractal Characteristics
}

\author{
Jie Yang, ${ }^{1}$ Yanna Zheng $\mathbb{D}^{1},{ }^{1}$ and Huijing Wang ${ }^{2}$ \\ ${ }^{1}$ School of Marine and Civil Engineering, Dalian Ocean University, Dalian 116023, China \\ ${ }^{2}$ Department of Naval Architecture, Dalian University of Technology, Dalian 116024, China
}

Correspondence should be addressed to Yanna Zheng; zhengyn@dlou.edu.cn

Received 15 December 2017; Accepted 31 January 2018; Published 24 April 2018

Academic Editor: Alain Portavoce

Copyright ( 2018 Jie Yang et al. This is an open access article distributed under the Creative Commons Attribution License, which permits unrestricted use, distribution, and reproduction in any medium, provided the original work is properly cited.

The damage process is accompanied by the acoustic emission for quasibrittle materials. And in the process of material damage evolution, the length of microcracks satisfies the fractal distribution. Research on their relationship in theory is helpful to reveal the law of material damage evolution and acoustic emission activities. Damage variable expressions are proposed based on the damage and fractal characteristics firstly. Then, the statistical models for acoustic emission considering damage and fractal characteristics are established by deducing the relationship between acoustic emission parameters and load cycles and fractal dimensions. The effects of damage and fractal effects on acoustic emission parameters are analyzed finally. The results show that the damage accelerates the $\mathrm{AE}$ activity to the rougher material, the opposite to the more homogeneous material. It can also be seen that the increase of the fractal dimension, the homogeneity constant $m$, will substantially increase the AE activities.

\section{Introduction}

Material fatigue damage is a dynamic process of complex plastic-cumulative damage accompanied by acoustic emission (AE) during damage degradation. The study of $\mathrm{AE}$ mechanisms provides a physical basis for interpretation of the behavior of acoustic emission which helps to understand the mechanism of fatigue fracture. Many efforts [1-4] have verified and identified the AE source mechanisms. Among them, the research on acoustic emission mechanism and acoustic emission characteristics based on micromechanics theory has always been paid more attention by researchers.

Tang and $\mathrm{Xu}[5,6]$ put forward the viewpoint that the damage variable is consistent with the acoustic emission parameters by the continuous damage mechanics method, and the statistical model of acoustic emission is established based on the Weibull distribution assuming damage evolution of each microelement defect. Analytical models [7] based on the continuous damage mechanics method have proven to be effective and have become a basis for numerical analysis related to the fracture process of materials [8].
Fatigue and fracture of the material is the result of damage accumulation. Internal damage to the structure during loading can cause stress redistribution, leading to new microdefect damage, and experimental studies $[9,10]$ have confirmed that the evolution of material damage has fractal characteristics; at the same time, fractal features and behaviors exist in the distribution of microcracks, the propagation of cracks and the evolution of material damage. On the other hand, AE behaviors during fracture are the reflection of the extent of damage depending on the evolution of internal defects and the mechanical process of reproduction in materials. It seems to suggest that the damage variables and constitutive equations are also responsible directly for the occurrence of AE in the material. However, the current statistical models of acoustic emission do not account for the effects of damage and fractal features on the constitutive equations of the materials, and the statistical models of acoustic emission are thus established without considering their effects.

In this paper, damage variables are redefined by AE parameters, the number of cycles, and the fractal dimension, and the expression of damage variable is 
corrected. The Weibull distribution [5] is further used to describe the damage evolution of microdefect, and then the material constitutive equation and load displacement equation considering damage and fractal characteristics are deduced to obtain the modified acoustic emission statistical model. Finally, the effects of damage and fractal characteristics on acoustic emission parameters are discussed.

\section{AE Parameters in Fatigue Fracture Process}

2.1. Damage Expressions of AE Parameters. Since an acoustic emission represents a minor damage of the material, as with stress and strain parameters, some AE parameters such as the event number of $\mathrm{AE}$ and the energy of $\mathrm{AE}$ are employed to the index of the damage degree. To investigate the relationship between the fatigue fracture process parameters and $\mathrm{AE}$ parameters, several definitions of damage variables are given as follows.

The material without initial damage under uniaxial loading is considered; assuming that the AE rate produced by per unit area of the microelement damage is $n_{\mathrm{v}}$, damage AE counts $\eta$ for the microelement area of $A_{\mathrm{d}}$ is as follows:

$$
\eta=n_{\mathrm{v}} \cdot A_{\mathrm{d}}
$$

where $A_{\mathrm{d}}$ is the cross-sectional area of microdefects area. The sum of ring down counts $\mathrm{AE}$ occurring until the total damage area of $A$ failure $\eta_{\mathrm{t}}$ is as follows:

$$
\eta_{\mathrm{t}}=n_{\mathrm{v}} \cdot A=\frac{\eta \cdot A}{A_{\mathrm{d}}} \text {. }
$$

Continuous damage variable $D$, characterized as the damage of material, has been introduced by Robotnov [11]:

$$
D=\frac{A_{\mathrm{d}}}{A}= \begin{cases}0 & \text { undamage state } \\ 1 & \text { damage state }\end{cases}
$$

where $A_{\mathrm{d}}$ is the cross-sectional area with damage and $A$ is the cross-sectional area without damage.

Combining (2) with (3), the relationship between the damage variable and $\mathrm{AE}$ count is obtained by

$$
D=\frac{\eta}{\eta_{\mathrm{t}}}
$$

It is shown that the $\mathrm{AE}$ count ratio can characterize the degree of damage evolution of the material, which is the macroscopic representation of the microscopic change, namely, the AE parameters that are consistent with the damage variables.

\subsection{The Expressions of AE Parameters Based on Damage Effect} and Fractal Characteristics. Damage variables can track the development of the crack in real time, and the fractal dimension can describe the crack shape quantitatively. If parameters both damage variables and fractal dimension are used to describe the damage evolution of the material, the influence of material defects on the mechanical behavior parameters can be further studied in a microscale.
Starting from the constitutive equation of the damage variable, the damage variable expression is modified, and the relationship between the damage variable defined by acoustic emission parameters and the new damage variable defined by damage and fractal characteristics is studied.

Fatigue damage is mainly caused by plastic accumulation and hardening properties for low cycle fatigue (LCF). Constitutive equations [12] derived from the damage variable $D$ for the low cycle fatigue are characterized as

$$
\dot{D}=\frac{\sigma^{m-1}}{B^{\prime}(\Delta \sigma)^{\lambda} N^{(1-\alpha)}(1-D)^{m}} \dot{\sigma}(\mathrm{LCF}),
$$

where $m=2 s_{0}+1 / n$ and $B^{\prime}=n G^{1 / n}\left(2 E S_{0}\right)^{s_{0}}$ are material constants and $s_{0}$ and $S_{0}$ are parameters. Damage constitutive equation for LCF is introduced by hardening state variable $G, G=G_{0} h$; assuming $G$ with power exponent functions $\Delta \sigma$ and $N, G=G_{0} h=G_{0}\left(\Delta \sigma^{\lambda} N^{(1-\alpha)}\right)^{n}$. Integrating (5) in one loading cycle, damage evolution equations $d D / d N$ can be obtained as

$$
\frac{d D}{d N}=\frac{(\Delta \sigma)^{m} h^{-1 / n}}{B^{\prime \prime}(1-D)^{m}}(\mathrm{LCF}),
$$

where $B^{\prime \prime}=m B^{\prime} / 2^{(m+1)}$. By integrating (6), under boundary conditions (if $N=0, D=0$, else $N=N_{\mathrm{f}}, D=1$ ), damage variable expressions derived from LCF can be given by

$$
D=1-\left(1-\left(\frac{N_{1}}{N_{\mathrm{f}}}\right)^{\alpha}\right)^{1 /(m+1)} .
$$

With $\alpha=0$, the above equations have the same patterns. So, (7) can be regarded as the general expression of the fatigue damage variable, which is similar to the results as discussed by Chaboche et al. [13].

When the material is subjected to fatigue, damage caused by external load, the fatigue damage variable $\mu$ after $N$ cycles could be defined as

$$
\mu=\frac{N}{N_{\mathrm{f}}},
$$

where $N_{\mathrm{f}}$ is the fatigue failure lifetime. Substituting (8) into (7), then

$$
1-(1-D)^{m+1}=\mu^{a}
$$

Substituting (4) and (8) into (9), the damage variable expression with damage can be described as

$$
1-\left(1-\frac{\eta}{\eta_{\mathrm{t}}}\right)^{m_{1}+1}=\left(\frac{N}{N_{\mathrm{f}}}\right)^{\alpha} .
$$

The above two relationships demonstrate that AE activities are related to fatigue damage.

According to the fracture surface fractal characteristics, the damage variable with damage and fractal characteristics in (9) is defined by Xie and Ju [14] as follows:

$$
\mu\left(D_{\mathrm{f}}, \delta\right)=\mu \delta^{D_{\mathrm{f}}-2}=1-(1-\mu) \delta^{D_{\mathrm{f}}-2},
$$

where $D_{\mathrm{f}}$ and $\widetilde{D}_{\mathrm{f}}$ are the fractal dimensions of the damage domain and the damage residual domain, respectively. 
Substituting (4) and (11) into (9), damage variable expression with fractal characteristics can be described as

$$
1-\left[1-\left(\frac{\eta}{\eta_{\mathrm{t}}}\right)\right]^{m+1}=\left[\left(\frac{N}{N_{\mathrm{f}}}\right) \delta^{D_{\mathrm{f}}-2}\right]^{\alpha} .
$$

The above-modified expressions ((10) and (12)) demonstrate that the damage can be defined by the number of loading cycles and the fractal dimension and also can be defined by acoustic emission parameters in AE caused by fatigue fracture. The theoretical results indicated that the $\mathrm{AE}$ parameters are related to the damage effect and fractal characteristics, so AE parameters can be used to evaluate the $\mathrm{AE}$ activity at different fatigue fracture stages.

\section{Modifications of AE Statistical Models Based on Damage and Fractal Characteristics}

The main contributor to damage is the rate of strain energy density release and is always associated with irreversible strain at the microscopic or macroscopic level. More generally, the damage rate is described by deformation variables such as the strain $\varepsilon$ of the following form [7]:

$$
d D= \begin{cases}f(\varepsilon) d \varepsilon & (\varepsilon=\zeta \text { and } \mathrm{d} \varepsilon>0) \\ 0 & (\varepsilon<\zeta \text { and } \mathrm{d} \varepsilon<0)\end{cases}
$$

where $f(\varepsilon)$ is a continuous positive definite function of $\varepsilon$, namely, the damage evolution function, and $\zeta$ is the variable damage strain threshold value. Assuming that the damage process of material is continuous, the Weibull distribution function is used to describe the damage evolution of these defects in this paper. In what follows, we will express the $\mathrm{AE}$ parameters by statistical method according to the AE parameters are consistent with and the damage variables.

It is assumed that the microunit strength of the materials obeys the Weibull distribution:

$$
\begin{aligned}
f(\varepsilon) & =\frac{m}{\varepsilon_{0}}\left(\frac{\varepsilon}{\varepsilon_{0}}\right)^{m-1} \exp \left[-\left(\frac{\varepsilon}{\varepsilon_{0}}\right)^{m}\right] \\
\text { or } f\left(\frac{u}{L}\right) & =\frac{m L}{u_{0}}\left(\frac{u}{u_{0}}\right)^{m-1} \exp \left[-\left(\frac{u}{u_{0}}\right)^{m}\right], \\
F(\varepsilon) & =1-\exp \left[-\left(\frac{\varepsilon}{\varepsilon_{0}}\right)^{m}\right] \\
\text { or } F\left(\frac{u}{L}\right) & =1-\exp \left[-\left(\frac{u}{u_{0}}\right)^{m}\right]
\end{aligned}
$$

where $u_{0}$ and $\varepsilon_{0}$ are the reference mean displacement and strain when the specimen reaches the peak load, respectively $\left(u_{0}=L \varepsilon_{0}\right)$; the displacement-strain relationship in one dimension is $u=L \varepsilon$, where $L$ is the length of the specimen and $m$ is the material heterogeneity (the larger the value, the more uniform the material).

For monotonic loading, the first expression in (13) is always established. The initial damage condition is considered, namely, $D=\varepsilon=\zeta=0$, and in terms of (4), (13), and (15), the cumulative number of $\mathrm{AE}$ can be expressed as

$$
\frac{\eta}{\eta_{\mathrm{t}}}=D=F(\varepsilon)=\int_{0}^{\varepsilon} f(x) d x=1-\exp \left[-\left(\frac{\varepsilon}{\varepsilon_{0}}\right)^{m}\right] .
$$

Here, we intend to show that the modified constitutive equations and load expressions can be proposed by a suitably chosen damage variable with damage and fractal characteristics.

3.1. Modifications of Constitutive Equations and Load Expressions with Damage Effect and Fractal Characteristics. Combining the effective stress concept [11] with (10), (14), and (15), the improved constitutive equation with damage of the material is expressed by the $\mathrm{AE}$ parameter as follows:

$$
\begin{aligned}
\sigma & =E \varepsilon(1-\mu)=E \varepsilon\left\{1-\left[1-\left(1-\frac{\eta}{\eta_{\mathrm{t}}}\right)^{m_{1}+1}\right]^{1 / \alpha}\right\} \\
& =E \varepsilon\left\langle 1-\left\{1-\exp \left[-\left(m_{1}+1\right)\left(\frac{\varepsilon}{\varepsilon_{0}}\right)^{m}\right]\right\}^{1 / \alpha}\right\rangle,
\end{aligned}
$$

where $\sigma$ is the stress, $E$ is the elastic modulus, and $m_{1}$ and $\alpha$ are the material constants.

The relation between the load $R$ and deformation $u$ with damage is obtained by

$$
\begin{aligned}
R & =g(u)=\frac{E A}{L}\left\langle 1-\left\{1-[1-F(\varepsilon)]^{m_{1}+1}\right\}^{1 / \alpha}\right\rangle u \\
& =k_{0} u\left\langle 1-\left\{1-\left[1-F\left(\frac{u}{L}\right)\right]^{m_{1}+1}\right\}^{1 / \alpha}\right\rangle \\
& =k_{0} u\left\langle 1-\left\{1-\exp \left[-\left(m_{1}+1\right)\left(\frac{u}{u_{0}}\right)^{m}\right]\right\}^{1 / \alpha}\right\rangle,
\end{aligned}
$$

where $k_{0}$ is the specimen of initial stiffness, $k_{0}=E A / L$; for the weakened properties of materials, namely, $\sigma^{\prime}\left(\varepsilon_{0}\right)<0$, then $m>1$.

In terms of (12), (14), and (15), the modified constitutive equation with fractal characteristics of the material is expressed by the AE parameter as follows:

$$
\begin{aligned}
\sigma & =\bar{\sigma}(1-\widetilde{\mu})=E \varepsilon\left(1-\mu \delta^{D_{\mathrm{f}}-2}\right)=E \varepsilon\left(1-\frac{N}{N_{\mathrm{f}}} \delta^{D_{\mathrm{f}}-2}\right) \\
& =E \varepsilon\left\{1-\delta^{D_{\mathrm{f}}-2}\left[1-\left(1-\frac{\eta}{\eta_{\mathrm{t}}}\right)^{m_{1}+1}\right]^{1 / \alpha}\right\} \\
& =E \varepsilon\left\langle 1-\delta^{D_{\mathrm{f}}-2}\left\{1-\exp \left[-\left(m_{1}+1\right)\left(\frac{\varepsilon}{\varepsilon_{0}}\right)^{m}\right]\right\}^{1 / \alpha}\right\rangle .
\end{aligned}
$$

The relation between the load $R$ and deformation $u$ with fractal characteristics is obtained by 


$$
\begin{aligned}
R & =g(u)=k_{0} u\left\langle 1-\delta^{D_{\mathrm{f}}-2}\left\{1-\left(1-F\left(\frac{u}{L}\right)\right)^{m_{1}+1}\right\}^{1 / \alpha}\right\rangle \\
& =k_{0} u\left\langle 1-\delta^{D_{\mathrm{f}}-2}\left\{1-\exp \left[-\left(m_{1}+1\right)\left(\frac{u}{u_{0}}\right)^{m}\right]\right\}^{1 / \alpha}\right\rangle .
\end{aligned}
$$

3.2. Modifications of AE Statistical Model Based on Damage Effect. The following expressions of AE rates are modified according to the constitutive equations and load expressions with damage and fractal characteristics in two cases.

In one case, loaded at displacement rate $c_{1}$ as a constant, the deformation rate of the specimen is given by

$$
\dot{u}=\frac{c_{1}}{1+g^{\prime}(u) / K_{\mathrm{m}}},
$$

where $K_{\mathrm{m}}$ is the load stiffness of the test machine. The expression of the AE rate with damage can be described as

$n_{\mathrm{t}}=\frac{c_{1}}{L} \frac{f(u / L)}{1+g^{\prime}(u) / K_{\mathrm{m}}}=\frac{m c_{1}}{u_{0}}$

$\frac{\left(u / u_{0}\right)^{m-1} \exp \left[-\left(u / u_{0}\right)^{m}\right]}{1+\left(k_{0} / K_{\mathrm{m}}\right)\left\lfloor\left\langle 1-\left\{1-\exp \left[-\left(m_{1}+1\right)\left(u / u_{0}\right)^{m}\right]\right\}^{1 / \alpha}\right\rangle-\left(\left(\left(m_{1}+1\right) m\right) / \alpha\right)\left(u / u_{0}\right)^{m}\left\{1-\exp \left[-\left(m_{1}+1\right)\left(u / u_{0}\right)^{m}\right]\right\}^{1 /(\alpha-1)} \exp \left[-\left(m_{1}+1\right)\left(u / u_{0}\right)^{m}\right]\right\rfloor}$.

The relationship between the deformation and the time of the specimen is obtained by

$$
\begin{aligned}
t & =\frac{1}{c_{1}}\left(u+\frac{g(u)}{K_{\mathrm{m}}}\right) \\
& \left.=\frac{u}{c_{1}} \mid 1+\frac{k_{0}}{K_{\mathrm{m}}}\left\langle 1-\left\{1-\exp \left[-\left(m_{1}+1\right)\left(\frac{u}{u_{0}}\right)^{m}\right]\right\}^{1 / \alpha}\right\rangle\right] .
\end{aligned}
$$

In the other case, loaded at stress increase rate $c_{2}$ as aconstant, the deformation rate of the specimen is given by

$$
\dot{u}=\frac{c_{2}}{g^{\prime}(u)} .
$$

The expression of the AE rate with damage can be described as

$$
\begin{aligned}
n_{t}= & \frac{c_{2} f(u / L)}{L g^{\prime}(u)}=\frac{m c_{2}}{k_{0} u_{0}} \\
& \cdot \frac{\left(u / u_{0}\right)^{m-1} \exp \left[-\left(u / u_{0}\right)^{m}\right]}{\left.\left\lfloor\left\langle 1-\left\{1-\exp \left[-\left(m_{1}+1\right)\left(u / u_{0}\right)^{m}\right]\right\}^{1 / \alpha}\right\rangle-\left(\left(m_{1}+1\right) m\right) / \alpha\right)\left(u / u_{0}\right)^{m}\left\{1-\exp \left[-\left(m_{1}+1\right)\left(u / u_{0}\right)^{m}\right]\right\}^{1 /(\alpha-1)} \exp \left[-\left(m_{1}+1\right)\left(u / u_{0}\right)^{m}\right]\right\rfloor} .
\end{aligned}
$$

The relationship between the deformation and the time of the specimen is obtained by

$$
t=\frac{g(u)}{c_{2}}=\frac{k_{0}}{c_{2}} u\left\langle 1-\left\{1-\exp \left[-\left(m_{1}+1\right)\left(\frac{u}{u_{0}}\right)^{m}\right]\right\}^{1 / \alpha}\right\rangle
$$

3.3. Modification of AE Statistical Model Based on Fractal Characteristics. In what follows, the statistical analysis of AE models are investigated considering damage and fractal characteristics.

In one case, loaded at displacement rate $c_{1}$ as a constant, the expression of the $\mathrm{AE}$ rate with fractal characteristics can be described as

$$
\begin{aligned}
n_{\mathrm{t}} & =\frac{c_{1}}{L} \frac{f(u / L)}{1+g^{\prime}(u) / K_{\mathrm{m}}}=\frac{m c_{1}}{u_{0}} \\
& \cdot \frac{\left(u / u_{0}\right)^{m-1} \exp \left[-\left(u / u_{0}\right)^{m}\right]}{1+\left(k_{0} / K_{\mathrm{m}}\right)\left\lfloor\left\langle 1-\delta^{D_{\mathrm{f}}-2}\left\{1-\exp \left[-\left(m_{1}+1\right)\left(u / u_{0}\right)^{m}\right]\right\}^{1 / \alpha}\right\rangle-\delta^{D_{f}-2}\left(\left(\left(m_{1}+1\right) m\right) / \alpha\right)\left(u / u_{0}\right)^{m}\left\{1-\exp \left[-\left(m_{1}+1\right)\left(u / u_{0}\right)^{m}\right]\right\}^{1 /(\alpha-1)} \exp \left[-\left(m_{1}+1\right)\left(u / u_{0}\right)^{m}\right]\right]} .
\end{aligned}
$$




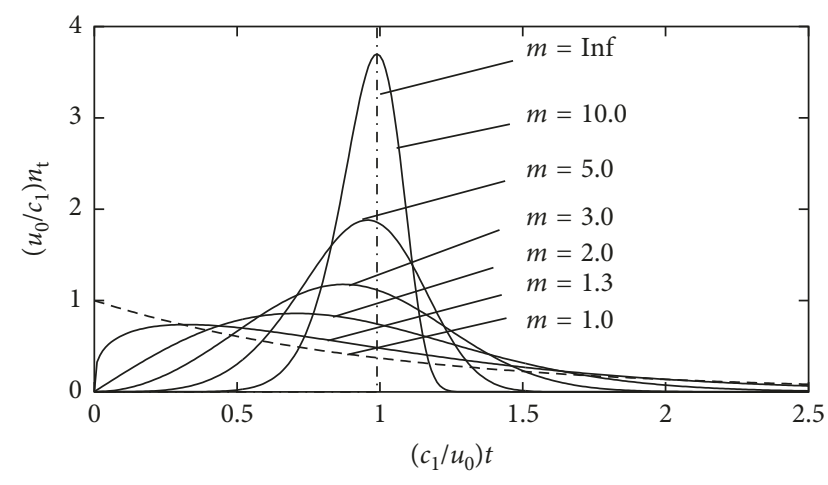

Figure 1: Relationship between AE rates $\left(u_{0} / c_{1}\right) n_{t}$ versus $\left(c_{1} / u_{0}\right) t$ of the material with homogeneity $m$ loaded at displacement rate $c_{1}$ as a constant with $K_{\mathrm{m}} \rightarrow \infty$ of the testing machine.

The relationship between the deformation and the time of the specimen is obtained by

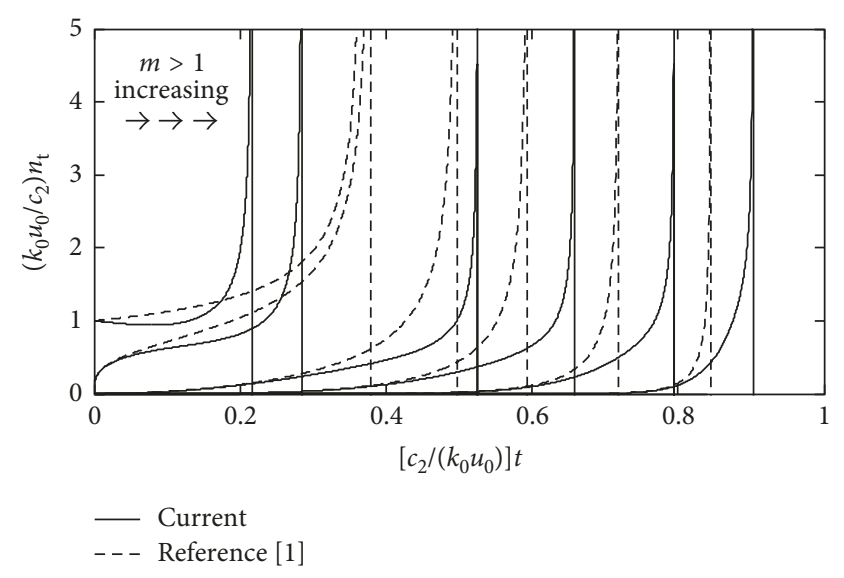

Figure 2: AE hit rates $\left(k_{0} u_{0} / c_{2}\right) n_{t}$ versus $\left[c_{2} /\left(k_{0} u_{0}\right)\right] t$ of the material with $m$ for constant stress increase rate $c_{2}$.

$$
\left.t=\frac{1}{c_{1}}\left(u+\frac{g(u)}{K_{\mathrm{m}}}\right)=\frac{u}{c_{1}} \mid 1+\frac{k_{0}}{K_{\mathrm{m}}}\left\langle 1-\delta^{D_{\mathrm{f}}-2}\left\{1-\exp \left[-\left(m_{1}+1\right)\left(\frac{u}{u_{0}}\right)^{m}\right]\right\}^{1 / \alpha}\right\rangle\right]
$$

In the other case, loaded at stress increase rate $c_{2}$ as a constant, the expression of the $\mathrm{AE}$ rate with fractal characteristics can be described as

$$
\begin{aligned}
n_{\mathrm{t}} & =\frac{c_{2} f(u / L)}{L g^{\prime}(u)}=\frac{m c_{2}}{k_{0} u_{0}} \\
& \cdot \frac{\left(u / u_{0}\right)^{m-1} \exp \left[-\left(u / u_{0}\right)^{m}\right]}{\left.L\left\langle 1-\delta^{D_{\mathrm{f}}-2}\left\{1-\exp \left[-\left(m_{1}+1\right)\left(u / u_{0}\right)^{m}\right]\right\}^{1 / \alpha}\right\rangle-\delta^{D_{\mathrm{f}}-2}\left(\left(\left(m_{1}+1\right) m\right) / \alpha\right)\left(u / u_{0}\right)^{m}\left\{1-\exp \left[-\left(m_{1}+1\right)\left(u / u_{0}\right)^{m}\right]\right\}^{1 /(\alpha-1)} \exp \left[-\left(m_{1}+1\right)\left(u / u_{0}\right)^{m}\right]\right]} .
\end{aligned}
$$

The relationship between the deformation and the time of the specimen is obtained by

$$
\begin{aligned}
t & =\frac{g(u)}{c_{2}} \\
& =\frac{k_{0}}{c_{2}} u\left\langle 1-\delta^{D_{\mathrm{f}}-2}\left\{1-\exp \left[-\left(m_{1}+1\right)\left(\frac{u}{u_{0}}\right)^{m}\right]\right\}^{1 / \alpha}\right\rangle .
\end{aligned}
$$

\section{Damage and Fractal Characteristics Analysis and Effect on AE Parameters}

The effects of damage and fractal characteristics on $\mathrm{AE}$ parameters are discussed below. The results in Figure 1 show the curve of the $\mathrm{AE}$ rate $\left(u_{0} / c_{1}\right) n_{t}$ versus time $\left(c_{1} / u_{0}\right) t$ with different homogeneity $m$ of quasibrittle materials when considering the initial stiffness ratio $K_{\mathrm{m}} \rightarrow \infty$ of the system.
There are three cases: the first one does not consider the damage, and the result of the AE rate is taken from Zhang et al. [7]; the second one considers the damage, and the calculation formula of the AE rate can be seen from (22) and (23); and the third considers the fractal damage, and the calculation formula of the AE rate can be seen from (27) and (28).

From Figure 1, it can be seen that the AE rate curves overlap when $m$ takes different values; that is, the values of the $\mathrm{AE}$ rate are identical for the three cases. The results show that the effects of damage/fractal effects on the AE parameters can be neglected when the initial stiffness is greater than $K_{\mathrm{m}}$ to infinity.

The change of $\mathrm{AE}$ rates related with different homogeneity $m$ for constant stress increase rate $c_{2}$ is presented in Figure 2. The dotted line represents the case without damage, and the calculation formula of acoustic emissivity is taken from [7]. The solid line represents the case with damage, the result from (25) and (26). It can be seen that the AE activity does not appear aftershock during the deformation process, 


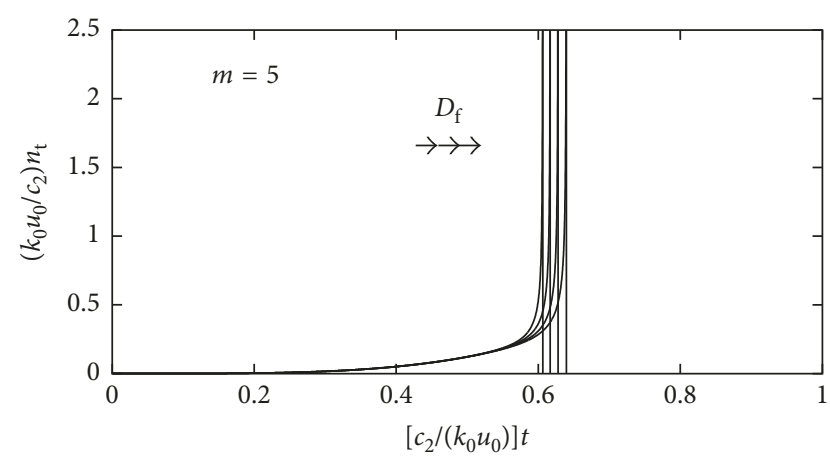

Figure 3: AE hit rates $\left(k_{0} u_{0} / c_{2}\right) n_{t}$ versus time $\left[c_{2} /\left(k_{0} u_{0}\right)\right] t$ of the material with various $D_{\mathrm{f}}$ for constant stress increase rate $c_{2}$.

and when the material becomes unstable and the main rupture occurs in the state of ultimate strength, the AE rates approach infinity. The rougher the material, the more obvious the precursor of main shock of AE activity. The damage accelerates the $\mathrm{AE}$ activity to the rougher material, the opposite to the more homogeneous material.

As shown in Figure 3, with $m_{1}=4 ; \alpha=0.2 ; m=5$; $\delta=0.5$, loaded at stress increase rate $c_{2}$ as a constant, the AE rate $n_{t}$ relates to time $t$ with different fractal dimension $D_{\mathrm{f}}$. It is also shown that the AE activity will increase with the increase in the fractal dimension $D_{\mathrm{f}}$.

\section{Conclusions}

Modifications of the AE statistical models based on the damage and fractal characteristics are presented, and the effects of damage and fractal effects on acoustic emission parameters are analyzed. The following conclusions can be drawn:

(1) The expressions of damage variables taking into account damage and fractal characteristics are modified. The AE parameter, in addition to its dependence on the load cycles, is shown to be a function of the fractal dimension.

(2) The statistical analysis of the AE models are modified considering damage and fractal characteristics. The results show that for the constant stress increase rate, the damage accelerates the $\mathrm{AE}$ activity to the rougher material, the opposite to the more homogeneous material. It can also be seen that the increase of the fractal dimension, the homogeneity constant $m$, will substantially increase the AE activities.

\section{Conflicts of Interest}

The authors declare that they have no conflicts of interest.

\section{References}

[1] M. N. Bassim and M. Houssny-Eman, "Acoustic emission during the low cycle fatigue of AISI4340 steel," Materials Science and Engineering, vol. 68, no. 1, pp. 79-83, 1984.

[2] D. Fang and A. Berkovits, "Fatigue design model based on damage mechanisms revealed by acoustic emission measurements," Journal of Engineering Materials and Technology, vol. 117, no. 4, pp. 200-208, 1995.

[3] C. Barile, C. Casavola, G. Pappalettera, and C. Pappalettere, "Analysis of crack propagation in stainless steel by comparing acoustic emissions and infrared thermography data," Engineering Failure Analysis, vol. 69, pp. 35-42, 2016.

[4] A. Monti, A. El Mahi, Z. Jendli, and L. Guillaumat, "Mechanical behaviour and damage mechanisms analysis of a flax-fibre reinforced composite by acoustic emission," Composites Part A: Applied Science and Manufacturing, vol. 90, pp. 100-110, 2016.

[5] C. A. Tang and X. H. Xu, "Evolution and propagation of material defects and Kaiser effect function," Journal of Seismological Research, vol. 13, no. 2, pp. 203-213, 1990.

[6] Z. H. Chen and C. A. Tang, "Theoretical and experimental studies for Kaiser effect in rock," The Chinese Journal of Nonferrous Metals, vol. 7, no. 1, pp. 9-12, 1997.

[7] M. Zhang, Z. Li, Q. Yang, and X. Feng, "A damage model and statistical analysis of acoustic emission for quasi-brittle materials," Chinese Journal of Rock Mechanics and Engineering, vol. 25, pp. 2493-2501, 2006.

[8] C. A. Tang and X. H. Xu, "A cusp catastrophic model of rock unstable failure," Chinese Journal of Rock Mechanics and Engineering, vol. 9, no. 2, pp. 100-107, 1990.

[9] B. B. Mandelbrot, The Fractal Geometry of Nature, W. H. Freeman and Company, New York, NY, USA, 1982.

[10] K. Falconer, Fractal Geometry: Mathematical Foundations and Applications, Wiley, Chichester, UK, 1990.

[11] Y. N. Robotnov, "Creep ruptures," in Proceedings of the 12th International Congress of Applied Mechanics, M. Hetenyi and W. G. Vincenti, Eds., pp. 342-349, Standford-Springer-Verlag, Berlin, Germany, 1968.

[12] J. Lemaitre, "Formulation and identification of damage kinetic constitutive equations," in Continuum Damage Mechanics Theory and Applications, D. Krajcinovic and J. Lemaitre, Eds., pp. 37-89, Springer Verlag, New York, NY, USA, 1987.

[13] J. L. Chaboche, H. Kaczmarek, and P. Raine, "On the interaction of hardening and fatigue damage in the 316 stainless steel. Advances in fracture research," in Proceedings of the 5th International Conference on Fracture, pp. 1381-1393, Cannes, France, March 1981.

[14] P. H. Xie and Y. Ju, "A study of damage mechanics theory in fractional dimensional space," Chinese Journal of Theoretical and Applied Mechanics, vol. 31, no. 3, pp. 300-310, 1999.

\section{Acknowledgments}

This research was supported by the grant (no. 20170540105) from the Natural Science Foundation of Liaoning Province of China. 


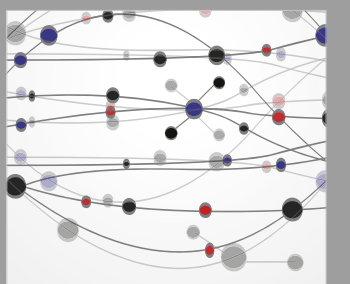

The Scientific World Journal
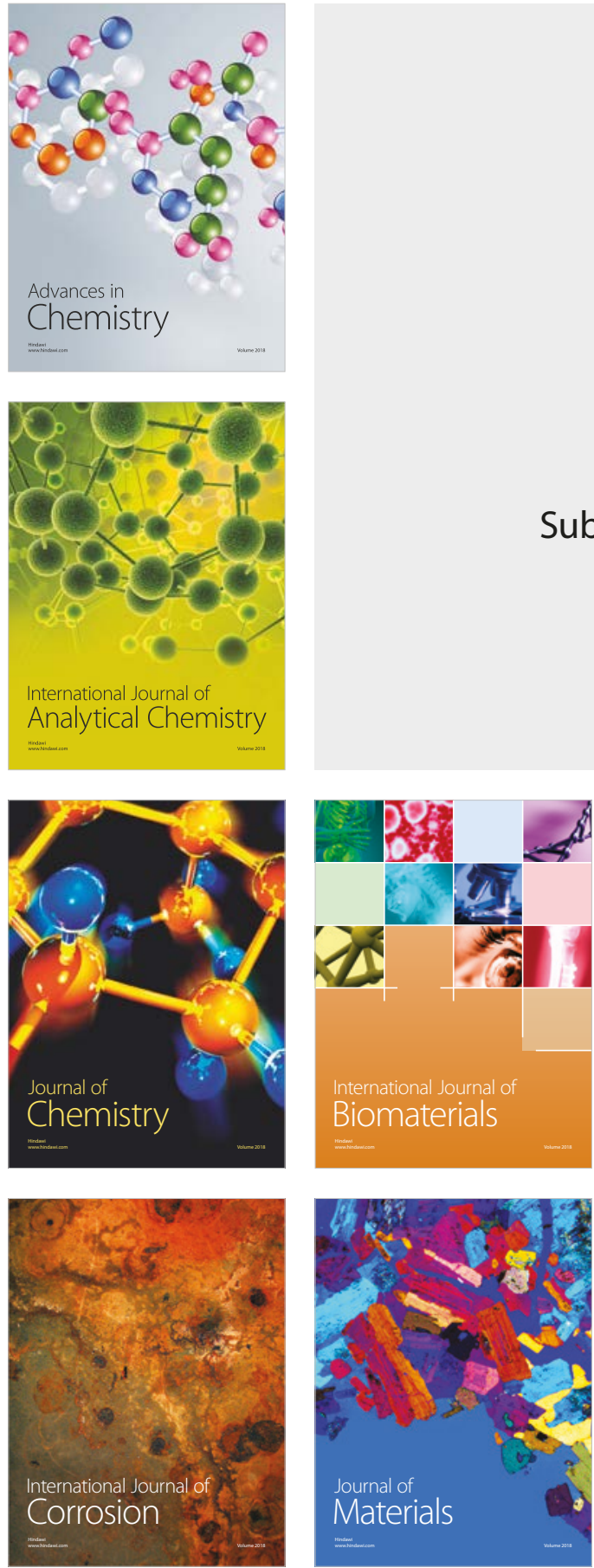

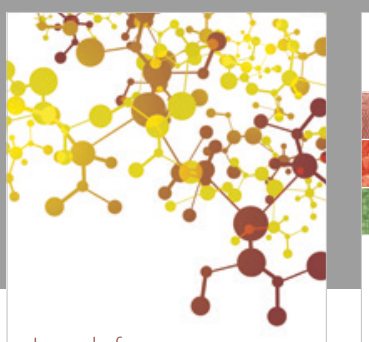

Journal of

Applied Chemistry
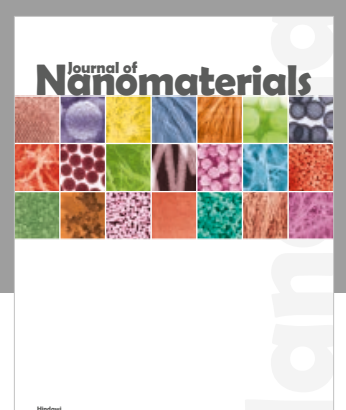

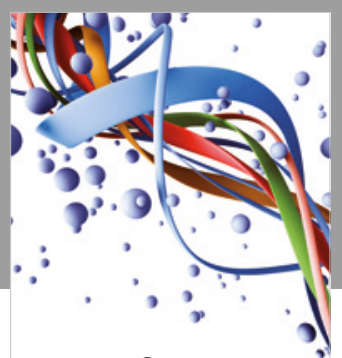

Scientifica

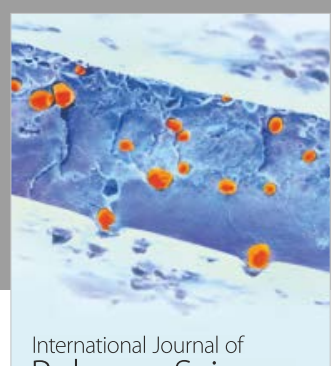

Polymer Science

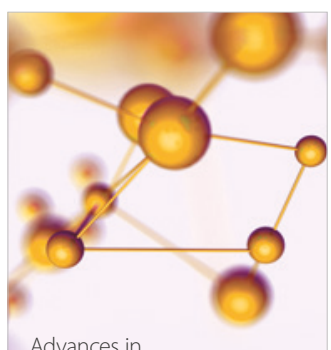

Physical Chemistry
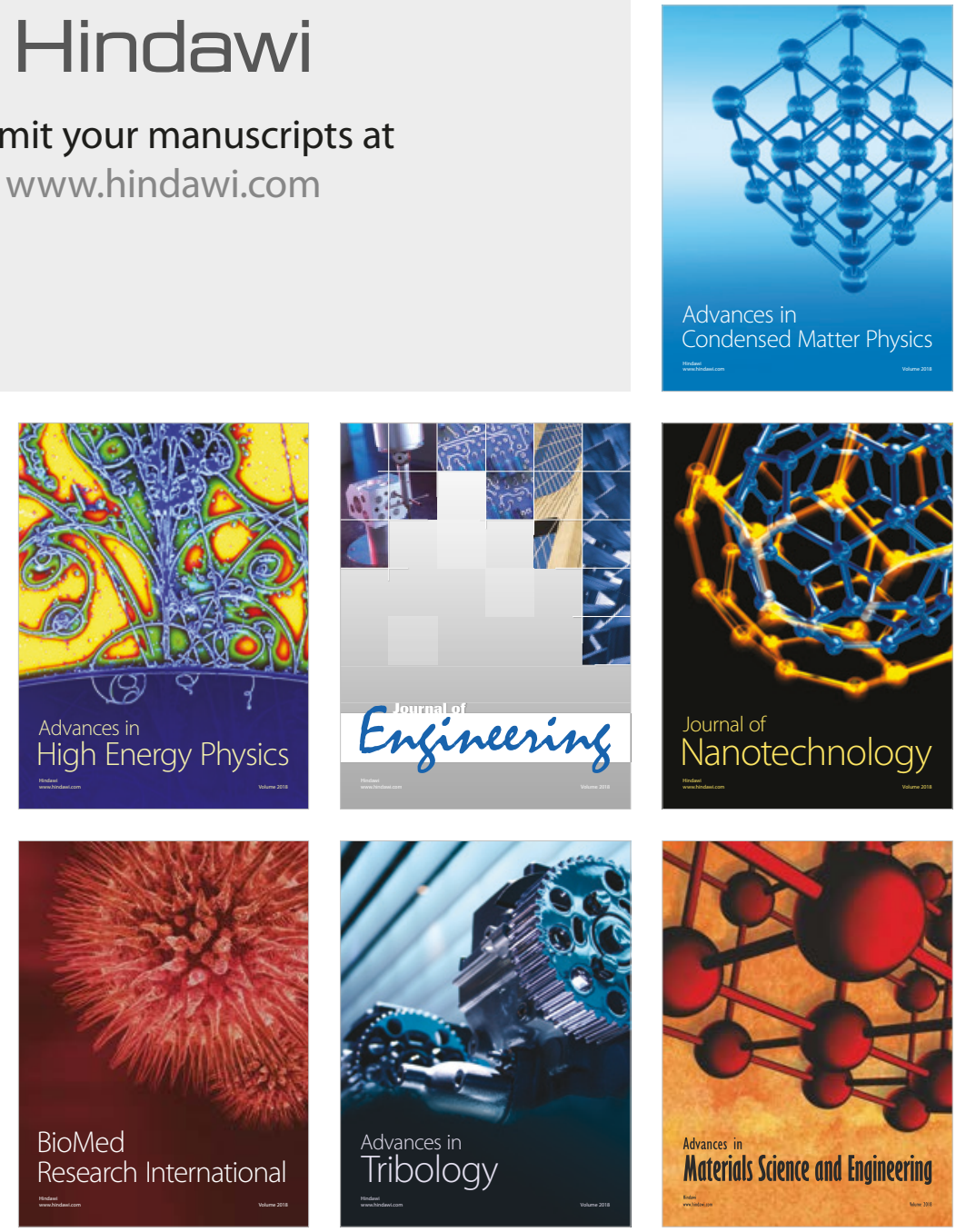